\title{
A nemaline myopathy mutation in $\alpha$-tropomyosin causes defective regulation of striated muscle force production
}

\author{
Daniel E. Michele, Faris P. Albayya, and Joseph M. Metzger \\ Department of Physiology, University of Michigan, Ann Arbor, Michigan 48109, USA \\ Address correspondence to: Joseph M. Metzger, 7730 Medical Science Building II, Department of Physiology, \\ University of Michigan, 1150 W. Medical Center Drive, Ann Arbor, Michigan 48109-0622, USA. \\ Phone: (734) 763-5844, Fax: (734) 936-8813; E-mail: metzgerj@umich.edu.
}

Received for publication July 13, 1999, and accepted in revised form October 25, 1999.

\begin{abstract}
Nemaline myopathy (NM) is a rare autosomal dominant skeletal muscle myopathy characterized by severe muscle weakness and the subsequent appearance of nemaline rods within the muscle fibers. Recently, a missense mutation inTPM3, which encodes the slow skeletal $\alpha$-tropomyosin $(\alpha \mathrm{Tm})$, was linked to NM in a large kindred with an autosomal-dominant, childhood-onset form of the disease. We used adenoviral gene transfer to fully differentiated rat adult myocytes in vitro to determine the effects of NM mutant human $\alpha \mathrm{Tm}$ expression on striated muscle sarcomeric structure and contractile function. The mutant $\alpha \mathrm{Tm}$ was expressed and incorporated correctly into sarcomeres of adult muscle cells. The primary defect caused by expression of the mutant $\alpha \mathrm{Tm}$ was a decrease in the sensitivity of contraction to activating $\mathrm{Ca}^{2+}$, which could help explain the hypotonia seen in NM. Interestingly, NM mutant $\alpha \mathrm{Tm}$ expression did not directly result in nemaline rod formation, which suggests that rod formation is secondary to contractile dysfunction and that load-dependent processes are likely involved in nemaline rod formation in vivo.
\end{abstract}

J. Clin. Invest. 104:1575-1581 (1999).

\section{Introduction}

Nemaline myopathy (NM) is clinically and genetically heterogeneous with congenital and childhood- and adult-onset forms, with at least 2 genetic loci identified (1). In its most dramatic form, NM is associated with severe striated muscle weakness at birth, more commonly referred to as a "floppy infant" phenotype. The etiology of NM and the mechanisms behind muscle weakness and nemaline rod formation are currently unknown. Although the hallmark NM rods, distributed within the sarcomere continuous with the Z-line, are composed of the $Z$-line component $\alpha$-actinin (2), this protein has been excluded to date as a candidate gene for NM. Recently, an M9R mutation was identified in tropomyosin $(\mathrm{Tm})(3)$, which is a key regulatory protein of the muscle thin filament as it relays signals from the $\mathrm{Ca}^{2+}$ sensor, the troponin complex, to the actin-myosin interactions that produce force $(4,5)$. There have to our knowledge been no prior studies addressing the biochemical, structural, or physiological effects of the NM mutation on $\alpha \mathrm{Tm}$ structure-function. Thus, how this specific point mutation in Tm may alter muscle function or results in specific aberrant sarcomeric structures such as nemaline rods is also unknown.

The mutated Met in TPM3 $\alpha \mathrm{Tm}$ associated with NM, as well as the entire $\mathrm{NH}_{2}$-terminus of $\alpha \mathrm{Tm}$, is highly conserved in $\mathrm{Tm}$ proteins from vertebrates to Caenorbabditis elegans and likely plays an important role in the structure-function of Tm (Figure 1a). The M9R mutation would also be predicted to disrupt the coiled- coil dimeric structure of $\mathrm{Tm}$, as this substitution places a charged amino acid in the "a" position of the heptad repeat (Figure 1b), a position that is hydrophobic in the coiled-coil consensus motif and is considered a forbidden site for charged amino acids (6).

The development and implementation of a fully differentiated muscle model system to directly test the effects of normal and mutant Tm on contractile function have been a key obstacle in understanding $\alpha \mathrm{Tm}$ structure and function. For example, successful extraction and reconstitution protocols in permeabilized muscle fibers, which have been used to substitute normal and engineered mutant troponin C proteins (7), are not available for $\mathrm{Tm}$. We have recently developed a rat adult cardiac myocyte primary culture system in which the highly differentiated state of the adult striated muscle cell is retained for at least 7 days in primary culture $(8,9)$. In addition, we have used adenoviral-mediated gene transfer into these fully differentiated muscle cells to replace the endogenous rat $\alpha \mathrm{Tm}$ with the human adult heart $\alpha \mathrm{Tm}$ isoform with a $\mathrm{COOH}$-terminal FLAG epitope without any deleterious effects on $\mathrm{Ca}^{2+}$-activated isometric force production (10). In the present study, we have used this system to express the NM mutation in $\alpha \mathrm{Tm}$ in fully differentiated muscle cells and, for the first time to our knowledge, tested the direct effects of this mutant $\alpha \mathrm{Tm}$ on muscle cell sarcomeric structure and contractile function. More specifically, we addressed the following questions: (a) Can $\alpha$ Tm with the NM mutation be expressed in striated muscle cells and 
incorporate into the muscle sarcomere? (b) Does NM mutant $\alpha$ Tm expression directly alter $\alpha$ Tm's regulation of striated muscle contractile function? (c) Does NM mutant $\alpha$ Tm mutation directly result in alterations in striated muscle structure (i.e., lead to the formation of nemaline rods) in vitro, or is nemaline rod formation a secondary consequence of the disease?

\section{Methods}

Generation of recombinant adenovirus. Replication-deficient recombinant adenoviral vectors were generated by homologous recombination in HEK-293 cells. The analogous M9R mutant Tm was generated by Quickchange mutagenesis (Stratagene, La Jolla, California, USA) of $\mathrm{Met}_{8}$ of the full-length human fast skeletal $\alpha$ Tm cDNA (11) with a COOH-terminal FLAG epitope and was verified by DNA sequencing. The $\alpha$ TmFLAG cDNA's were subcloned into pCA4 shuttle vector and cotransfected with pJM17 into HEK-293 cells (8). Recombinant adenoviral vectors were plaque purified and screened by restriction enzymes and Southern blot analysis. Plaque purified adenoviral vectors were grown to high titer (1 $\times 10^{10}$ plaque-forming units [PFU] per milliliter) and purified by $\mathrm{CsCl}$ centrifugation.

Isolation of adult cardiac myocytes. The heart was removed from an anesthetized, female Sprague Dawley rat (200 $\mathrm{g}$ ), and single ventricular cardiac myocytes were isolated by collagenase-hyaluronidase digestion as described previously $(8,9)$. The myocytes were then infected with recombinant adenoviral vectors at approximately 300 500 moi (PFU/cell) diluted in DMEM, serum free with penicillin/streptomycin $(\mathrm{P} / \mathrm{S})$. The myocytes were maintained in DMEM, serum free, $\mathrm{P} / \mathrm{S}$ throughout the time in culture. In a subset of experiments, myocytes were stimulated at $0.5 \mathrm{~Hz}$ in a custom electrical field stimulation chamber in Media 199 (Sigma Chemical Co., St. Louis, Missouri, USA) supplemented with $5 \mathrm{mM}$ glutathione, $0.2 \mathrm{mg} / \mathrm{mL} \mathrm{BSA}$, and $\mathrm{P} / \mathrm{S}$.

Analysis of protein expression. Total myofilament protein expression was analyzed by scraping myocytes off of 18$\mathrm{mm}^{2}$ coverslips and into SDS-PAGE sample buffer followed by SDS-PAGE and Western blot analysis as described previously $(8,10)$. To measure whether or not the expressed Tm was bound to the myofilaments, protein samples were prepared from myocytes on coverslips that were permeabilized in relaxing solution (see later here) with $0.1 \%$ Triton $\mathrm{X}-100$ in relaxing solution (Pierce Chemical Co., Rockford, Illinois, USA) for 5-10 seconds and the myocytes were washed in several 1-minute washes of fresh relaxing solution before collection for Western blot analysis. This protocol is similar to that used for permeabilizing myocytes for isometric force recordings shown below and, therefore, is sufficient to disrupt the isolated cardiac myocyte membrane.

Indirect immunofluorescence, confocal microscopy, and electron microscopy. Indirect immunofluorescence was carried out as described previously (8). The primary antibody for detection of $\alpha$ TmFLAG was M2 (Sigma Chemical Co.; 1:500). Myocytes were dual labeled with FITC phalloidin
(Sigma Chemical Co.; $1 \mu \mathrm{g} / \mathrm{mL}$ ) for 20 minutes. Immunofluorescence was viewed with a Nikon Diaphot $200 \mathrm{mi}$ croscope (Nikon Inc., Melville, New York, USA) outfitted with a NORAN confocal laser imaging system (NORAN Instruments, Middleton, Wisconsin, USA).Electron microscopy of cardiac myocytes was performed at day 5 after gene transfer with and without electrical stimulation as described previously (12).

Single cardiac myocyte functional analysis. Relaxing and activating solutions (13) contained (in mmol/L): 7 EGTA, 1 free $\mathrm{Mg}^{2+}$, 4 MgATP, 14.5 creatine phosphate, 20 imidazole, and sufficient $\mathrm{KCl}$ to yield a total ionic strength of $180 \mathrm{mmol} / \mathrm{L}$. Solution $\mathrm{pH}$ was adjusted to 7.0 with $\mathrm{KOH}$. The $\mathrm{pCa}\left(\right.$ i.e., $-\log \left[\mathrm{Ca}^{2+}\right]$ ) of the relaxing solution was set at 9.0, and the $\mathrm{pCa}$ of the maximal activating solution was 4.0. Intermediate $\mathrm{pCa}$ solutions were generated by mixing the $\mathrm{pCa} 9.0$ and $\mathrm{pCa} 4.0$ solutions. To characterize the direct relationship between steady-state force production and $\left[\mathrm{Ca}^{2+}\right]$, cardiac myocytes were permeabilized with $0.1 \%$ Triton $\mathrm{X}-100$, a nonionic detergent, in relaxing solution for 5-10 seconds followed by several washes of relaxing solution. This allows direct access of the bathing solutions to the intact myofilaments of the cardiac myocyte and allows precise control of the intracellular ionic conditions including the $\left[\mathrm{Ca}^{2+}\right]$. Single, rod-shaped, permeabilized cardiac myocytes were attached to micropipettes coated with an adhesive between a force transducer (Model 403A; Cambridge Technology Inc., Cambridge, Massachusetts, USA) and moving coil galvanometer (Model 6350; Cambridge Technology Inc.) mounted on 3-way positioners. Sarcomere length was set at $2.2 \mu \mathrm{m}$. At each $\mathrm{pCa}$, steady-state isometric tension was allowed to develop, followed by rapid slackening to obtain the baseline tension. Total tension was measured as the difference in tension just before and after the slack step. Active tension was calculated by subtracting the resting tension (measured at pCa 9.0). Tension-pCa curves were fit using the Marquardt-Levenberg nonlinear least squares fitting algorithm and the Hill equation in the form: $P=\left[\mathrm{Ca}^{2+}\right]^{n} /\left(\mathrm{K}^{n}+\left[\mathrm{Ca}^{2+}\right]^{n}\right)$, where $P$ is the fraction of maximum tension $\left(\mathrm{P}_{\mathrm{o}}\right), K$ is the $\left[\mathrm{Ca}^{2+}\right]$ that yields one-half maximum tension, and $n$ is the Hill coefficient $\left(n_{\mathrm{H}}\right)$. ANOVA with a Student Neuman-Keuls post hoc test were used to examine significant differences, with $P<0.05$ indicating significance.

\section{Results}

We recently reported that expression and myofilament incorporation of the human $\alpha \mathrm{Tm}$ expressed in heart (TPM1) in isolated rat cardiac myocytes does not alter myocyte contractile function (10). In addition, there is high amino acid sequence identity ( $>90 \%$ for the entire protein), especially at the $\mathrm{NH}_{2}$-terminus, between human $\alpha$ Tm produced by the TPM1 gene and the diseased gene TPM3 (Figure 1a). With these 2 points in mind, we have introduced into TPM1 human $\alpha \mathrm{Tm}$, the analogous M9R mutation associated with NM (Figure 1a) to address the effects of the NM mutation on Tm 
Table 1

Summary of Western blot analysis of expression of normal and NM mutant $\alpha$ TmFLAG at days 5-6 after gene transfer in adult myocytes

\begin{tabular}{lccc}
\hline & $\begin{array}{c}\text { Intact } \\
\alpha \text { TmFLAG/ } \\
\text { Total Tm }(\%)\end{array}$ & $\begin{array}{c}\text { Perm } \\
\alpha \text { TmFLAG/ } \\
\text { Total Tm }(\%)^{*}\end{array}$ & $\begin{array}{c}\text { Total Tm/ } \\
\text { actin }\end{array}$ \\
Control & 0 & 0 & $1.00 \pm 0.08$ \\
& & & $(n=6)$ \\
Ad $\alpha$ TmFLAG & $43.3 \pm 1.4$ & $37.6 \pm 2.1$ & $1.18 \pm 0.11$ \\
300 moi & $(n=5)$ & $(n=7)$ & $(n=7)$ \\
Ad $\alpha$ TmFLAG M9R & $45.4 \pm 3.3$ & $39.5 \pm 2.7$ & $1.26 \pm 0.10$ \\
300 moi & $(n=8)$ & $(n=10)$ & $(n=6)$ \\
Ad $\alpha$ TmFLAG M9R & $42.9 \pm 4.0$ & $33.5 \pm 2.9$ & ND \\
150 moi & $(n=4)$ & $(n=6)$ & \\
& & &
\end{tabular}

* No permeabilized groups (perm) were statistically different than intact $(P>$ 0.05 ; $t$ test), and percent Tm incorporation into myofilaments was not statistically different in any of the groups $(P>0.05$; ANOVA). Total Tm/actin ratio was not statistically different in any of the groups $(P>0.05$; ANOVA). ND, not determined.

and its role in striated muscle structure and function. A $\mathrm{COOH}$-terminal-FLAG epitope was used to specifically distinguish the expressed $\mathrm{Tm}$ from the endogenous $\mathrm{Tm}$. Adult rat cardiac myocytes were isolated, treated with adenoviral vectors, and maintained for 5-6 days in serum-free media $(8,9)$. Figure 2 a shows the expression of normal and NM mutant $\alpha \mathrm{Tm}$ in adult myocytes at 5-6 days after gene transfer and illustrates several important points. First, NM mutant $\alpha \mathrm{Tm}$ can be expressed to similar levels as normal $\alpha \operatorname{Tm}(\sim 40 \%$ of total Tm), and the levels correlate well with the turnover rate of the endogenous Tm isoform ( 5.5 days; ref. 14) (Table 1). Second, permeabilization of the cardiac myocytes before sampling produced no significant change in the ratio of the expressed normal or NM mutant $\alpha \mathrm{Tm}$ to the total Tm (Table 1). If the expressed Tm was not incorporating into myofilaments, permeabilization of the cardiac myocyte membrane would be expected to remove and reduce or eliminate the newly expressed $\alpha \mathrm{Tm}$ proteins. Because there was no effect of permeabilization on the expressed $\alpha T \mathrm{~m}$ protein content based on the Western analysis (Table 1), this indirectly indicates that the expressed NM mutant $\alpha \mathrm{Tm}$ can bind to the myofilaments in a similar manner as does the expressed normal $\alpha \mathrm{Tm}$. Finally, the expression of normal and NM mutant $\alpha \mathrm{Tm}$ produced no significant changes in Tm stoichiometry (Table 1; calculated as the total $\mathrm{Tm} /$ actin in each lane) or the pattern of isoform expression of the endogenous $\mathrm{Tm}$ and the other thin filament regulatory proteins troponin $\mathrm{T}$ and troponin I (Figure 2a). These results indicate the only regulatory protein alteration detected in the myocytes expressing ectopic $\mathrm{Tm}$ is the replacement of the endogenous $\mathrm{Tm}$ protein without nonspecific effects resulting from gene transfer or ectopic $\mathrm{Tm}$ protein expression on the differentiated state of the adult myocytes.

To assess more directly the incorporation of the expressed NM mutant $\alpha \mathrm{Tm}$ into sarcomeres of adult muscle cells, indirect immunofluorescence and confocal microscopy were used. Figure 2, b and c, shows that the immunohistochemistry labeling profiles for the ex-pressed epitope tagged normal and NM mutant Tm in adult myocytes have the periodic staining characteristic of striated muscle. It should be noted that the resting sarcomere length in cultured adult cardiac myocytes is about $1.8-1.9 \mu \mathrm{m}$, which results in the overlap of thin filaments. Therefore, the pattern of immunofluorescence would not be expected to show clearly segregated I-bands. Most importantly, the pattern of immunofluorescence of NM mutant $\alpha \mathrm{Tm}$ does not differ from the normal $\alpha \mathrm{Tm}$ pattern and is similar to our previous re-sults with expression of epitope-tagged $\alpha \mathrm{Tm}$ in adult cardiac myocytes (10). Because the epitope tag allows an exact and unique identification of the newly synthesized normal and mutant $\alpha \mathrm{Tm}$ proteins, the immunofluorescence confocal image analysis directly shows that the NM mutant $\alpha \mathrm{Tm}$ protein incorporates into the sarcomere similar to normal $\alpha \mathrm{Tm}$. Dual labeling of

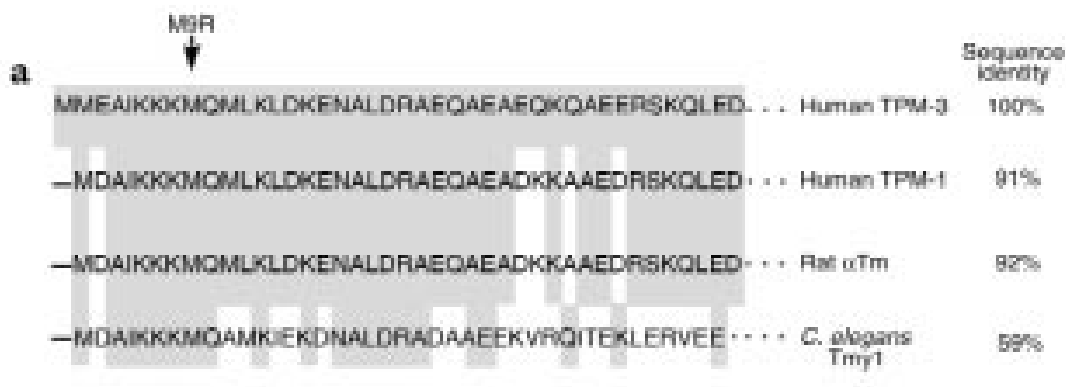

b

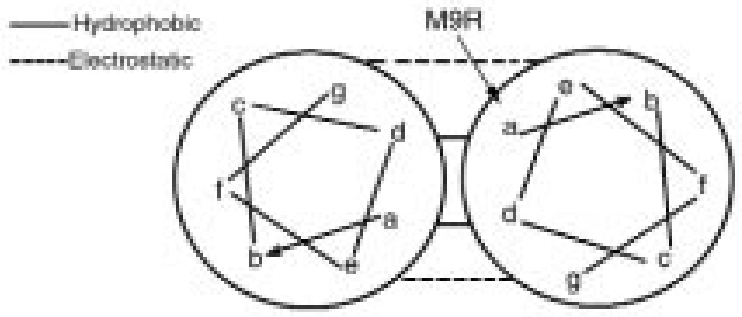

\section{Figure 1}

(a) Alignment of $\alpha \mathrm{Tm}$ isoforms. Genebank accession numbers are: TPM3, X04201; TPM1, M19713; rat, SEG_RATTMA; C. elegans., D38540. (b) Alpha-helical wheel diagram showing the predicted position (22) of the M9R mutation within the hydrophobic core "a" position of the coiled coil motif. 
a
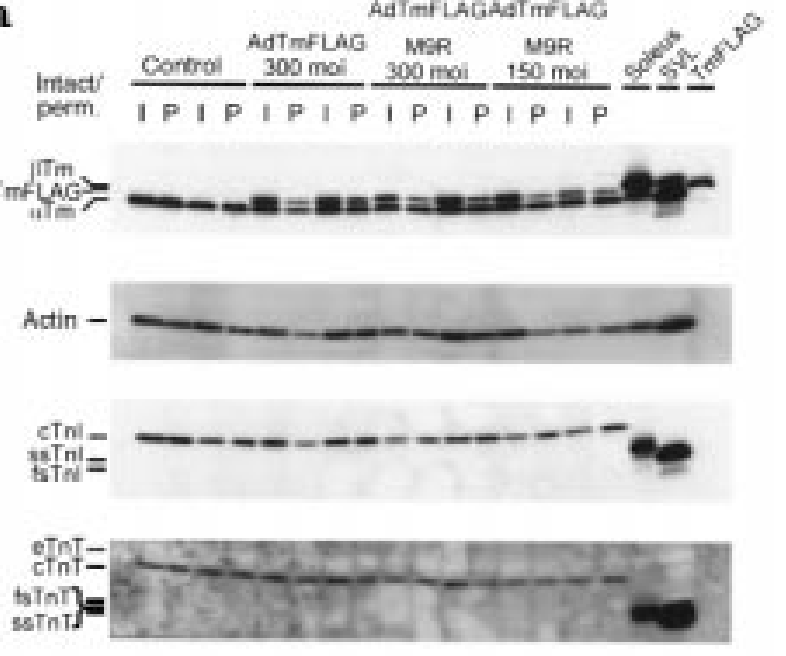

b

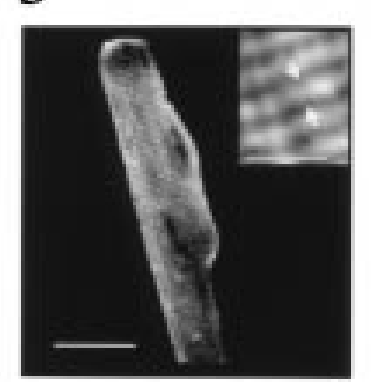

c

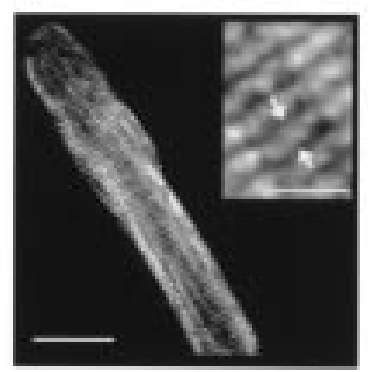

Figure 2

(a) Expression of thin filament proteins in adult myocytes expressing normal and NM mutant $\alpha \mathrm{Tm}$ at days 5-6 after gene transfer. Two different viral titers (300 and 150 moi, respectively) for Ad $\alpha$ TmFLAG M9R giving similar levels of expression were used in functional studies shown in Figure 4. Antibodies used in the panels from top to bottom were: Tm311 for Tm, 5C5 for sarcomeric actin, MAB1691 for troponin I, JLT-12 for troponin T. Soleus and superficial vastus lateralis were included to show migration of alternate myofilament protein isoforms. (b) Anti-FLAG immunofluorescence localization of the expressed epitope-tagged normal Tm ( $\alpha$ TmFLAG) in a representative adult myocyte 5 days after gene transfer. (c) AntiFLAG immunofluorescence localization of the expressed epitopetagged NM mutant Tm ( $\alpha$ TmFLAG M9R) in a representative adult myocyte 5 days after gene transfer. Insets in $\mathbf{b}$ and $\mathbf{c}$ show enlarged views of the striated anti-FLAG immunofluorescence staining in adult myocytes with arrowheads delineating the edges of the sarcomere (at each Z-line). Resting sarcomere length, $\sim 1.8-1.9 \mu \mathrm{m}$. At this sarcomere length, the thin filaments are overlapping, so segregated I-bands are not expected to be seen. Scale bars are $20 \mu \mathrm{m}$ for the panels and $4 \mu \mathrm{m}$ for the insets in $\mathbf{b}$ and $\mathbf{c}$.

cells with FITC phalloidin revealed no evidence of global changes in sarcomeric structure in cells expressing normal or mutant $\alpha \mathrm{Tm}$ (data not shown). Scoring of myocytes indicated that greater than $85 \%$ of all the rod-shaped cardiac myocytes were expressing normal or NM mutant $\alpha \mathrm{Tm}$ in each experimental group. Taken together, the immunolocalization results and the expression results suggest that NM mutant $\alpha \mathrm{Tm}$ can be expressed and incorporated normally into muscle sarcomeres.
Electron microscopy was used to assess the direct effect of NM mutant $\alpha \mathrm{Tm}$ expression on sarcomeric structure and to determine whether nemaline rod formation could be induced in vitro. Figure 3 , a-c, shows there were no detected effects of normal or NM mutant $\alpha \mathrm{Tm}$ expression on sarcomeric structure and no evidence of nemaline rod formation. Because these fully differentiated adult myocytes are quiescent when cultured in serum-free conditions, additional experiments were performed on myocytes electrically stimulated continuously at $0.5 \mathrm{~Hz}$ for 5 days in primary culture. Unloaded myocyte contraction did not cause any different effects of NM mutant $\alpha \mathrm{Tm}$ expression on sarcomeric structure or nemaline rod formation (Figure 3 , $\mathrm{d}-\mathrm{e})$. Therefore, NM mutant $\alpha \mathrm{Tm}$ expression and incorporation do not directly result in nemaline rod formation in striated adult muscle cells at least under these experimental conditions.

The effects of NM mutant Tm expression on the regulation of contraction was assessed by directly measuring isometric force production in single isolated adult myocytes. Importantly, Figure 4 shows that NM mutant Tm expression is associated with a marked decrease in the sensitivity of isometric force production to activating $\mathrm{Ca}^{2+}$. These results demonstrate for the first time to our knowledge that the primary defect in muscle expressing NM mutant $\mathrm{Tm}$ is altered regulation of force production. Interestingly, this primary defect in regulation of force production occurs in the absence of nemaline rod formation, raising the possibility that NM rod formation is a secondary phenomenon resulting from this defect. There were no detectable effects of normal or NM mutant $\alpha \mathrm{Tm}$ expression on the cooperativity of activation (Hill coefficient, $\mathrm{n}_{\mathrm{H}}$ ) or the maximal force production (Table 2). In addition, there was no significant difference in the $\mathrm{Ca}^{2+}$ sensitivity of isometric force production associated with normal $\alpha \mathrm{Tm}$ FLAG expression, indicating that the epitope tag does not directly alter $\alpha \mathrm{Tm}$ regulatory function as shown previously (10), and supporting our conclusion that altered contractile function is specifically due to the M9R mutation in $\alpha \mathrm{Tm}$.

\section{Discussion}

The M9R $\alpha \operatorname{Tm}$ mutation associated with NM is inherited in an autosomal dominant fashion $(1,3)$. Therefore, the possibility did exist that NM M9R Tm allele is a "null," meaning the protein is not expressed or not incorporated into sarcomeres, and the functional and structural alterations seen in NM patients are due to Tm haploinsufficiency. However, the results presented here directly demonstrate that NM mutant Tm can be expressed and incorporated normally into striated muscle sarcomeres (Figure 2). These results showing expression and myofilament incorporation of NM mutant $\alpha \mathrm{Tm}$ suggest that NM does not result from Tm insufficiency, but rather indicate the NM mutant $\alpha$ Tm exerts a dominant negative effect by directly altering $\mathrm{Tm}$ regulation of contractile structure/function. 


\section{Table 2}

Lack of effect of NM mutant Tm expression on $\mathrm{Ca}^{2+}$-activated maximal force generation $\left(P_{0}\right)$ and myofilament cooperativity $\left(n_{H}\right)$. Ad $\alpha$ TmFLAG M9R treated myocytes were not statistically different than control or Ad $\alpha$ TmFLAG-treated myocytes $(P>0.05)$.

\begin{tabular}{|c|c|c|}
\hline & $\mathrm{P}_{0}\left(\mathrm{kN} / \mathrm{m}^{2}\right)$ & Hill coefficient $\left(n_{H}\right)$ \\
\hline Control & $\begin{array}{c}13.6 \pm 2.4 \\
(n=11)\end{array}$ & $\begin{array}{c}1.50 \pm 0.14 \\
(n=10)\end{array}$ \\
\hline Ad $\alpha \operatorname{TmFLAG~}$ & $\begin{array}{c}11.4 \pm 2.1 \\
(n=8)\end{array}$ & $\begin{array}{c}1.80 \pm 0.13 \\
(n=9)\end{array}$ \\
\hline Ad $\alpha \operatorname{TmFLAG~M9R}$ & $\begin{array}{c}15.9 \pm 1.7 \\
(n=23)\end{array}$ & $\begin{array}{c}1.73 \pm 0.09 \\
(n=23)\end{array}$ \\
\hline
\end{tabular}

The most striking result of the present study was that expression of $\alpha \mathrm{Tm}$ containing the NM mutation had direct effects on $\mathrm{Ca}^{2+}$-activated isometric force production that could help explain the hypotonia seen in NM. Although maximal force was unchanged, the effect of NM mutant Tm expression reduced the amount of force produced at submaximal activating $\mathrm{Ca}^{2+}$ concentrations. It has been estimated that the peak $\left[\mathrm{Ca}^{2+}\right]_{\mathrm{i}}$ in a single twitch in amphibian and mammalian muscle fibers is approximately $5 \mu \mathrm{M}$ (pCa 5.3), whereas maximal $\left[\mathrm{Ca}^{2+}\right]_{\mathrm{i}}$ during tetanus is approximately $10 \mu \mathrm{M}$ (pCa 5.0) $(15,16)$. However, molecular modeling of $\mathrm{Ca}^{2+}$ transients and binding to $\mathrm{Ca}^{2+}$ binding proteins suggest that, owing to the short duration of the $\mathrm{Ca}^{2+}$ transient, the troponin complex is only approximately $70 \%$ saturated by $\mathrm{Ca}^{2+}$ during a twitch (17), and the troponin saturation decays before maximal twitch force is developed $(18,19)$. Indeed, $x$-ray diffraction results from intact frog skeletal muscle at temperatures greater than $14^{\circ} \mathrm{C}$ indicate $\mathrm{Tm}$ movement and thin filament activation are not complete during a twitch contraction (20). Together, these results suggest that skeletal muscle may operate at submaximal thin filament activation levels under physiological conditions. Therefore, we hypothesize that the observed decrease in myofilament sensitivity to activating $\mathrm{Ca}^{2+}$ (Figure 4) would directly contribute to reduced force production and muscle weakness seen in NM .

Little is known about the role of specific amino acids in $\mathrm{Tm}$ involved in the $\mathrm{Ca}^{2+}$ regulation of the thin filament. The effects of the M9R mutation on Tm regulatory function suggest that coiled-coil interactions between $2 \mathrm{Tm}$ monomers near the $\mathrm{NH}_{2}$-terminus are critical for normal $\mathrm{Tm}$ regulatory function. Interestingly, the $\mathrm{NH}_{2}$-terminal 9 amino acids are critical for Tm binding to actin (21) and for head-tail interactions of Tm that polymerizes along the entire length of the thin filament (22). The $\mathrm{NH}_{2}$-terminus of Tm also contains a binding site for the $\mathrm{NH}_{2}$-terminus of troponin $\mathrm{T}$ subunit of the troponin complex (23), and therefore M9R may disrupt the signaling pathway from the $\mathrm{Ca}^{2+}$ binding subunit troponin $\mathrm{C}$, leading to alterations in myofilament $\mathrm{Ca}^{2+}$ sensitivity. Although the present results do suggest that disrupting the coiled coil dimer near the $\mathrm{NH}_{2}$-terminus of Tm can directly alter the sensitivity of
$\mathrm{Ca}^{2+}$-activated force production, further experiments are necessary to determine whether the NM mutation in Tm exerts its effects through altered interactions with actin, the troponin complex, or the actin-myosin interaction. It may then be possible to use appropriate $\mathrm{Ca}^{2+}$ sensitizers (that exert their effect through these interactions) to reverse the effects of NM mutant Tm expression on the $\mathrm{Ca}^{2+}$ sensitivity of isometric force production.

The direct desensitization of $\mathrm{Ca}^{2+}$ activated force production caused by NM mutant $\alpha \mathrm{Tm}$ is different than the effect of expression of a mutant $\alpha \mathrm{Tm}$ associated with familial hypertrophic cardiomyopathy (FHC), which recently has been shown to produce hypersensitization of $\mathrm{Ca}^{2+}$-activated force production in cardiac muscle from transgenic mice (24). The FHC mutation, D175N, resides near a region that is proposed to interact with the $\mathrm{COOH}$-terminus of troponin $\mathrm{T}$ suggesting that interactions of $\mathrm{Tm}$ with differing regions of troponin $\mathrm{T}$ may produce different effects on myofilament $\mathrm{Ca}^{2+}$ sensitivity. It is also possible that the FHC and NM mutations differentially affect the conformation of Tm such that the FHC mutation favors a conformation allowing myosin interactions with actin, and the NM mutation favors a conformation that blocks myosin interactions with actin, resulting in the changes in $\left[\mathrm{Ca}^{2+}\right]$
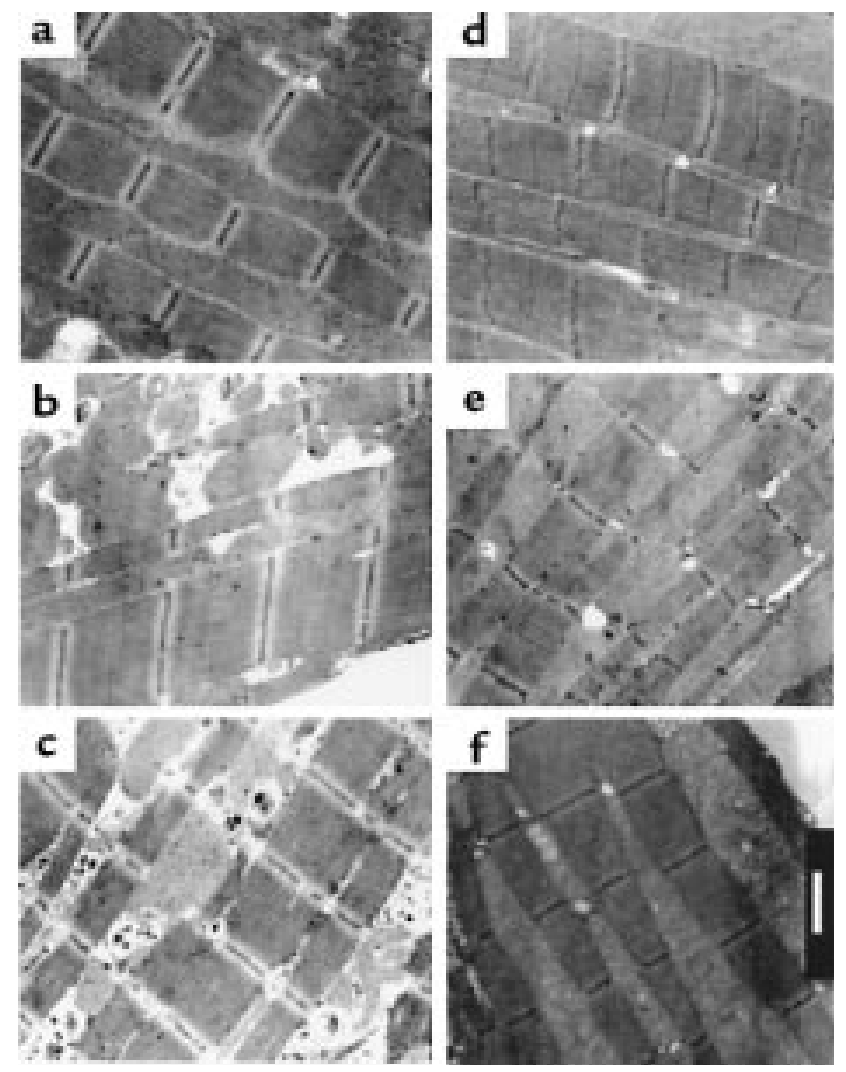

Figure 3

Electron micrographs of day 5 quiescent adult myocytes (a-c) or day 5 adult myocytes contracting at $0.5 \mathrm{~Hz}(\mathbf{d}-\mathbf{f})$. The representative myocytes shown were treated with no vector $(\mathbf{a}, \mathbf{d})$, Ad $\alpha \mathrm{TmFLAG}(\mathbf{b}$, e), or Ad $\alpha$ TmFLAG M9R (c, f). Scale bar is $1 \mu \mathrm{m}$. 
a

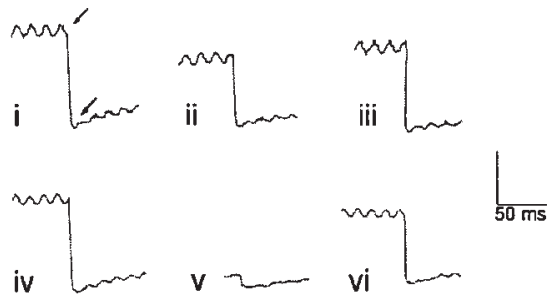

b

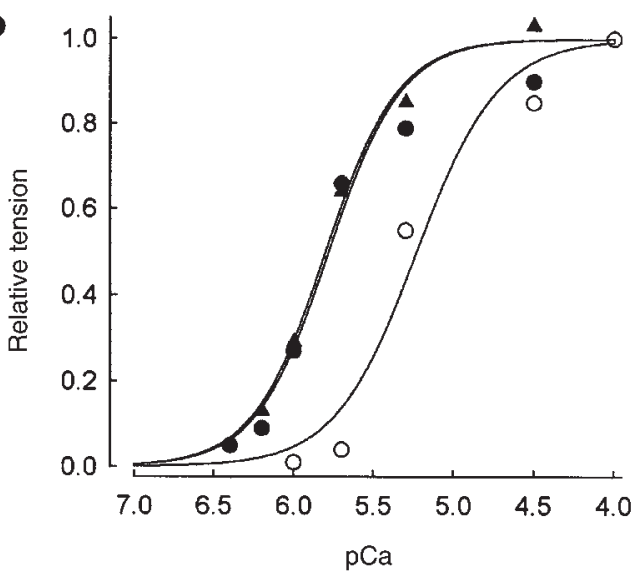

C

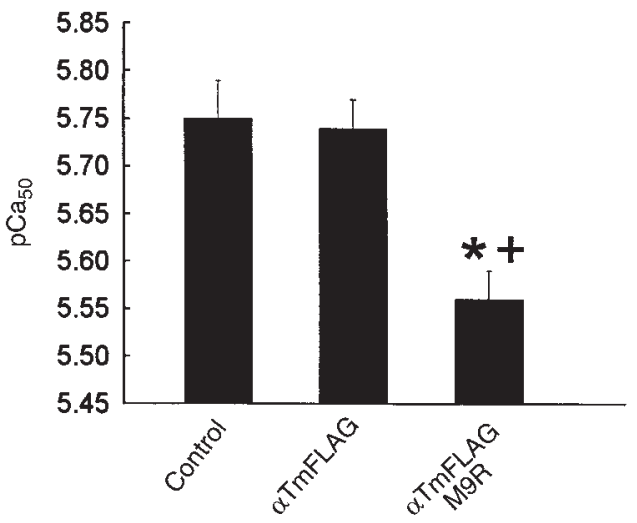

Figure 4

Steady-state isometric force production in adult myocytes expressing normal and NM mutant $\alpha \mathrm{Tm}$. (a) Original fast time-based force recordings from single adult myocytes expressing normal $\alpha T m$ (i-iii) or NM mutant $\alpha \mathrm{Tm}$ (iv-vi) using maximal ( $\mathrm{pCa} 4.0, \mathrm{i}$, iii, iv, vi) or submaximal (pCa 5.7, ii, v) $\mathrm{Ca}^{2+}$-activating solutions. Arrows depict the slack step of the myocyte for calculation of active tension production. (b) Summary of tension-pCa relationship of a representative control myocyte (triangles) and a myocyte expressing normal $\alpha \mathrm{Tm}$ (closed circles) or NM mutant Tm (open circles). (c) Summary of the activating $\left[\mathrm{Ca}^{2+}\right]$ required for half-maximal activation $\left(\mathrm{pCa}_{50}\right)$ in control adult myocytes $(n=10)$ and expressing normal $(n=9)$ and NM mutant $\alpha \operatorname{Tm}(n=23) .{ }^{*}$ Significantly different from control $(P<0.05$; ANOVA). + Significantly different from $\alpha \operatorname{TmFLAG~}(P<0.05$; ANOVA).

required for activation of the thin filament. Interestingly, the FHC mutant Tm is expressed in cardiac and skeletal muscle but does not produce a noted skeletal muscle myopathy (25). This supports our proposal here that the desensitization of $\mathrm{Ca}^{2+}$-activated force production is specific to NM associated with the mutation in $\alpha \mathrm{Tm}$, and this functional alteration may underlie the NM mutant $\alpha \mathrm{Tm}$-associated skeletal muscle myopathy.

Interestingly, $\mathrm{NM}$ mutant $\alpha \mathrm{Tm}$ expression and incorporation caused contractile dysfunction in the absence of formation of nemaline rods in quiescent striated muscle cells or myocytes undergoing unloaded contractions (Figure 3). On the basis of these results, we propose that nemaline rod formation is a secondary event in the pathogenesis of NM. One possibility is that the mutant $\alpha \mathrm{Tm}$ functionally weakens sarcomeres in series, which could result in loaddependent sarcomere damage over time, inappropriate sarcomere remodeling, and progressive nemaline rod formation in vivo. This hypothesis is supported clinically, as many patients with NM show clinical phenotypes (e.g., hypotonia) before nemaline rods are visible, and rods are seen only when a subsequent, second muscle biopsy is analyzed (1). In addition, other unrelated conditions, such as HIV infection and hemodialysis in the absence of mutant sarcomeric protein expression, have been shown to induce nemaline rods in muscle fibers $(26,27)$. Further, nemaline rods are also seen in combination with other types of skeletal muscle myopathies such as central core disease and mitochondrial myopathies (1). Taken together, these studies suggest that nemaline rod formation may be a shared secondary effect of multiple different pathologies or alterations in muscle function, use, or disuse. We believe that adult cardiac myocytes are an adequate model system to test the effects of NM mutant Tm on striated muscle sarcomere structure because some patients with NM do present a cardiomyopathy as well, with nemaline rods forming in both skeletal and cardiac muscle cells (28). This suggests that although $\mathrm{NM}$ is predominantly a skeletal muscle disorder, because the genes identified so far with mutations associated with NM (TPM3, ref. 3; and nebulin, ref. 29) are not expressed in cardiac muscle, nemaline rod formation can and does occur in cardiac muscle cells. Therefore, we hypothesize that nemaline rods are not directly produced by NM mutant Tm expression but are a secondary, load-dependent phenomenon associated with functional alterations caused by NM mutant $\mathrm{Tm}$. We cannot rule out that when nemaline rods do form, they contribute additional alterations of muscle contractile function. It is also possible that $\mathrm{NM} \mathrm{mu-}$ tant $\mathrm{Tm}$ directly plays a role in the formation of nemaline rods through interactions with other myofilament proteins, such as $\alpha$-actinin, but requires remodeling of the sarcomere in the presence of NM mutant $\alpha \mathrm{Tm}$ over periods longer than the 6-day culture period of the present experimental system.

Our study, which addresses the direct effects of NM mutant $\mathrm{Tm}$ on striated muscle structure-function, may also contribute to understanding the pathogenesis of mutations in many of the contractile proteins of the striated muscle sarcomere linked to several clinically important cardiac and skeletal myopathies. These 
include mutations in proteins involved in force generation, like the $\beta \mathrm{MyHC}$ mutations associated with hypertrophic cardiomyopathy and skeletal muscle central core disease $(30,31)$ and proteins involved in force transmission, as suggested by the recently identified actin mutation associated with dilated cardiomyopathy (32). In summary of our findings, NM results from the mutation in $\mathrm{Tm}$ that directly alters the $\mathrm{Ca}^{2+}$ regulation of force production. The focus of future scientific endeavors will be to identify how primary alterations in force generation, force transmission, and/or regulation of force production lead to the secondary, multifactorial phenotypes of myocyte hypertrophy, myocyte death, and/or sarcomeric structural alterations seen in diseases of striated muscle in vivo.

\section{Acknowledgments}

We thank Krystyna Pasyk for her excellent electron microscopy work, Margaret Westfall for her helpful discussion, and Clare Gooding for the gracious gift of the human $\alpha$ Tm cDNA. We also thank the Morphology and Image Analysis Core of the Michigan Diabetes Research and Training Center for use of its confocal microscope. This work was supported by grants from the National Institutes of Health (NIH) and the American Heart Association (AHA) (to J.M. Metzger) and NIH training grants to D.E. Michele. J.M. Metzger is an Established Investigator of the AHA.

1. North, K.N., et al. 1997. Nemaline myopathy: current concepts. J. Med. Genet. 34:705-713.

2. Jockusch, B.M., et al. 1980. Immunofluorescence microscopy of a myopathy. Alpha-actinin is a major constituent of nemaline rods. Exp. Cell Res. 127:409-420.

3. Laing, N.G., et al. 1995. A mutation in the $\alpha$-tropomyosin gene TPM3 associated with autosomal dominant nemaline myopathy. Nat. Genet. 9: 75-79.

4. Farah, C.S., and Reinach, F.C. 1995. The troponin complex and regulation of muscle contraction. FASEB J. 9:755-767.

5. Tobacman, L.S. 1996. Thin filament-mediated regulation of cardiac contraction. Annu. Rev. Physiol. 58:447-481.

6. Kohn, W.D., Mant, C.T., and Hodges, R.S. 1997. $\alpha$-Helical protein assembly motifs. J. Biol. Chem. 272:2583-2586.

7. Moss, R.L. 1992. $\mathrm{Ca}^{2+}$ regulation of mechanical properties of striated muscle: Mechanistic studies using extraction and replacement of regulatory proteins. Circ. Res. 70:865-884.

8. Westfall, M.V., Rust, E.M., Albayya, F., and Metzger, J.M. 1998. Adenovirus mediated myofilament gene transfer into adult cardiac myocytes. Methods Cell. Biol. 52:307-322.

9. Rust, E.M., Westfall, M.V., and Metzger, J.M. 1997. Stability of the contractile assembly and $\mathrm{Ca}^{2+}$-activated tension in adenovirus infected adult cardiac myocytes. Mol. Cell. Biochem. 181:143-155.
10. Michele, D.E., Albayya, F.P., and Metzger, J.M. 1999. Thin filament protein dynamics in fully differentiated adult cardiac myocytes: toward a model of sarcomere maintenance. J. Cell Biol. 145:1483-1495.

11. MacLeod, A.R., and Gooding, C. 1988. Human hTm $\alpha$ gene: expression in muscle and non-muscle tissue. Mol. Cell Biol. 8:433-440.

12. Metzger, J.M., Lin, W.I., and Samuelson, L.C. 1994. Transition in cardiac contractile sensitivity to calcium during the in vitro differentiation of mouse embryonic stem cells. J. Cell Biol. 126:701-711.

13. Metzger, J.M., et al. 1993. Skeletal troponin C reduces contractile sensitivity to acidosis in adult cardiac myocytes from transgenic mice. Proc. Natl. Acad. Sci. USA. 90:9036-9040.

14. Martin, A.F. 1981. Turnover of cardiac troponin subunits. J. Biol. Chem. 256:964-968.

15. Delbono, O., and Stefani, E. 1993. Calcium transients in single mammalian muscle fibers. J. Physiol. (Lond.). 463:689-707.

16. Caputo, C., Edman, K.A.P., Lou, F., and Sun, Y.-B. 1994. Variation in myoplasmic $\mathrm{Ca}^{2+}$ during contraction and relaxation studied by the indicator fluo-3 in frog muscle fibers. J. Physiol.(Lond.). 478:137-148.

17. Robertson, S.P., Johnson, J.D., and Potter, J.D. 1981. The time course of $\mathrm{Ca}^{2+}$ exchange with calmodulin, troponin, parvalbumin and myosin in response to transient increases in $\mathrm{Ca}^{2+}$. Biophys. J. 34:559-569.

18. Gillis, J.M. 1985. Relaxation of vertebrate skeletal muscle. A synthesis of biochemical and physiological approaches. Biochim. Biophys. Acta. 811:97-145.

19. Rüegg, J.C. 1986. Calcium in muscle activation. Springer-Verlag. Berlin, Germany. 83-113.

20. Kress, M., Huxley, H.E., Faruqi, A.R., and Hendrix, J. 1986. Structural changes during activation of frog skeletal muscle studied by time resolved X-ray diffraction. J. Mol. Biol. 188:325-342.

21. Cho, Y.-J., Liu, J., and Hitchcock-DeGregori, S.E. 1990. The amino terminus of muscle tropomyosin is a major determinant for function. $J$. Biol. Chem. 265:538-545.

22. McLachlan, A.D., and Stewart, M. 1975. Tropomyosin coiled-coil interactions: evidence for an unstaggered structure. J. Mol. Biol. 98:293-304.

23. Leavis, P.C., and Gergely, J. 1984. Thin filament proteins and thin-filament linked regulation of vertebrate muscle contraction. CRC Crit. Rev. Biochem. 16:235-305.

24. Muthuchamy, M., et al. 1999. Mouse model of a familial hypertrophic cardiomyopathy mutation in $\alpha$-tropomyosin manifests cardiac dysfunction. Circ. Res. 85:47-56.

25. Thierfelder, L., et al. 1994. $\alpha$-Tropomyosin and cardiac troponin T mutations cause familial hypertrophic cardiomyopathy: a disease of the sarcomere. Cell. 77:701-712.

26. Simpson, D.M., and Bender, A.N. 1988. Human immunodeficiency virus-associated myopathy: analysis of 11 patients. Ann. Neurol. 24:79-84.

27. Savica, V., et al. 1983. Plasma and muscle carnitine levels in haemodialysis patients with morphological-ultrastructural examination of muscle samples. Nephron. 35:232-236.

28. Ishibashi-Ueda, H., et al. 1990. Congenital nemaline myopathy with dilated cardiomyopathy. Hum. Pathol. 21:77-82.

29. Pelin, K., et al. 1999. Mutations in the nebulin gene associated with autosomal recessive nemaline myopathy. Proc. Natl. Acad. Sci. USA. 96:2305-2310.

30. Fananapazir, L., Dalakas, M.C., Cyran, F., Cohn, G., and Epstein, N.D. 1993. Missense mutations in the $\beta$-myosin heavy chain cause central core disease in hypertrophic cardiomyopathy. Proc. Natl. Acad. Sci. USA. 90:3993-3997.

31. Geisterfer-Lowrance, A.A.T., et al. 1996. A mouse model of familial hypertrophic cardiomyopathy. Science. 272:731-734.

32. Olson, T.M., Michels, V.V., Thibodeau, S.N., Tai, Y.-S., and Keating, M.T. 1998. Actin mutations in dilated cardiomyopathy, a heritable form of heart failure. Science. 280:750-752. 